\title{
A FOUNDATION FOR STRATEGIC AGENDA VOTING*
}

\author{
JOSE APESTEGUIA ${ }^{\dagger}$, MIGUEL A. BALLESTER ${ }^{\ddagger}$, AND YUSUFCAN MASATLIOGLU $^{\S}$
}

\begin{abstract}
We offer complete characterizations of the equilibrium outcomes of two prominent agenda voting institutions that are widely used in the democratic world: the amendment, also known as the Anglo-American procedure, and the successive, or equivalently the Euro-Latin procedure. Our axiomatic approach provides a proper understanding of these voting institutions, and allows comparisons between them, and with other voting procedures.
\end{abstract}

Keywords: Strategic Voting, Agendas, Committees, Institutions, Axioms.

JEL classification numbers: C72; D02; D71; D72.

\section{INTRODUCTION}

A proper understanding of the democratic institutions that are used in practice is a prime concern in the social sciences. In this paper we focus on two prominent voting procedures that are used extensively in parliamentary, legislative, and committee decision-making, world-wide: the amendment and the successive agenda procedures. While the amendment procedure is extensively used in the Anglo-American world, such as in the US Congress, the successive procedure is in place in many European countries as well as the European Parliament. In a nutshell, in this paper we offer for the first time foundations of these two procedures that enhance our understanding of these key voting institutions.

Date: February, 2012.

* We thank Salvador Barbera, Tilman Borgers, Chris Chambers, Matthew Jackson, Humberto Llavador, Ludovic Renou, and Arunava Sen for helpful comments. Financial support by the Spanish Commission of Science (ECO2008-04756, ECO2008-01768, ECO2009-12836 and ECO2010-09555-E), the Barcelona GSE research network, and the Government of Catalonia is gratefully acknowledged.

${ }^{\dagger}$ ICREA and Universitat Pompeu Fabra. E-mail: jose.apesteguia@upf .edu.

¥ Universitat Autonoma de Barcelona. E-mail: miguelangel.ballester@uab.es.

$\S$ University of Michigan. E-mail: yusufcan@umich.edu. 
Both, the amendment and the successive procedures are voting institutions used to collectively select one alternative from a set of alternatives. They represent natural extensions of simple majority voting to cases where there are more than two alternatives. In both cases, the alternatives are ordered, forming an agenda, and are considered sequentially, taking at each step in the sequence binary decisions using majority voting. In the particular case of the amendment procedure, two alternatives are jointly considered at each step, and the binary choice consists in deciding by majority voting which alternative is eliminated, and hence which alternative is confronted with the next one in the agenda. For the sake of illustration consider three alternatives, ordered as $(a, b, c)$. Then in this case $a$ is voted against $b$, and the winner against $c$. The winner of this last confrontation is declared elected. See Figure 1(a) for a graphical representation.

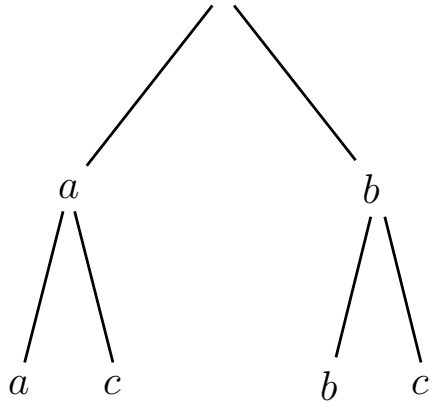

(a) Amendment procedure

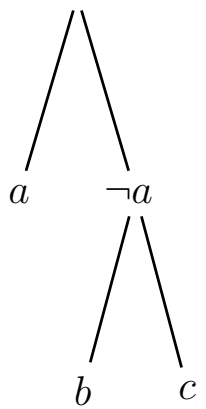

(b) Successive procedure

FiguRE 1. Structure of amendment procedure and successive procedure with the agenda $(a, b, c)$.

In the successive procedure, an alternative is considered at each step in the sequence, and the binary choice made by majority voting is whether to select it, or to reject it, and in the event of rejection, to consider the next alternative in the sequence. For instance, in the above example, voters must in fact decide between accepting $a$ or rejecting it, in which case they confront the problem of selecting an alternative from $\{b, c\}$. If they prefer $a$ to the alternatives in $\{b, c\}$ then the voting ends. Otherwise they compare the next alternative in the sequence, which is $b$, with the remaining one, alternative $c$. Figure $1(\mathrm{~b})$ provides a graphical representation. 
It turns out that versions of these voting procedures are extensively used in committee decision-making, as well as in parliamentary institutions world-wide. Moreover, there seems to be a geographical concentration of the type of agenda voting institution in use. While the amendment procedure is prevalent in the Anglo-American world, European countries adopt the successive procedure. See Table 1 for an illustration. ${ }^{1}$

\begin{tabular}{c|c}
\hline \hline Amendment & Successive \\
\hline Anglo-American & Euro-Latin \\
\hline USA & Austria, Belgium, Czech Republic, Denmark \\
Canada & France, Germany, Greece, Hungary, Iceland \\
UK & Ireland, Italy, Luxembourg, Netherlands \\
Sweden & Norway, Poland, Portugal \\
Finland & Slovakia, Slovenia, Spain \\
Switzerland & European Parliament \\
\hline \hline
\end{tabular}

TABLE 1. Agenda voting procedures by country.

Given, then, the practical importance of these voting procedures, it is not surprising that they have been subject to substantial theoretical and empirical research, that has led to the clarification of important aspects. Specifically, there is now a good understanding of the nature of the elected outcomes when voters vote either strategically or naively (see Farquharson, 1969; Miller, 1977, 1980; McKelvey and Niemi, 1978; Plott and Levine, 1978; Moulin, 1979, 1986; Shepsle and Weingast, 1984; Banks, 1985; Eckel and Holt, 1989; and Bag, Sabourian and Winter 2009). The effect of the agenda on the final elected outcome has also been the subject of intense research (see McKelvey, 1981; Shepsle and Weingast, 1982; Ferejohn, Morris Fiorina and McKelvey 1987; Dutta, Jackson and Le Breton, 2002; and Bernheim, Rangel and Rayo, 2006). Finally, there are papers that study which voting procedure maximizes in expectation the well-being of society (see Moser, 2007; see also Apesteguia, Ballester and Ferrer, 2011). These questions

\footnotetext{
${ }^{1}$ Black (1948) represents the first formal treatment of agenda voting institutions. Riker (1958) and Farquharson (1969) study the agenda voting procedures actually used in the US Congress. See also Shepsle and Weingast (1982), Ordeshook and Schwartz (1987), and Schwartz (2008). See Rasch (2000) for a treatment of the European case.
} 
are of prime importance for the understanding of the voting institutions with which we are concerned.

Here we take a different approach, and offer, for the first time, complete characterizations of these voting institutions that uniquely identify them. We establish the sets of properties that when imposed on a decision rule give the same outcome as the one obtained with strategic voters in the corresponding voting procedure. We show that the two procedures are characterized by two systems of three properties each, sharing a common intuition. The two systems of three properties are formed by (i) a Condorcet-type property, (ii) a property that guides the election in the presence of cycles generated by binary majority voting, and (iii) a consistency property imposing structure on the elections across related sets of alternatives. These characterizations allow a deep understanding of the properties satisfied by the voting institutions, and facilitate comparisons between them, and with other voting procedures. Furthermore, the identification of the characterizing properties of the voting institutions allows us to evaluate their normative appeal, and encourages the study of the consequences of relaxing or strengthening some of these properties. ${ }^{2}$

The first two properties are both Condorcet-type, but follow different directions. While in the case of the Euro-Latin procedure it is the classical Condorcet Consistency property, in the case of the Anglo-American procedure it is a Condorcet Loser Consistency property. That is, in the former case it is imposed that if there is an alternative that is preferred to every other alternative by a majority of voters, this is the alternative that should be selected. This is typically regarded as a fundamental property to be desired in any sensible voting procedure. In the Anglo-American case, on the other hand, the Condorcet property dictates that if there is an alternative that is ranked below every other alternative by a majority of voters, not only should this alternative not be elected, but it should not affect the final election either. This is clearly another desirable property.

The second type of properties discriminate between alternatives in the presence of cycles generated by majority voting. Cycles give rise to a difficult problem:

$\overline{2}$ In section 5.2 we illustrate the sort of voting institutions that emerge when we relax each of the characterization properties, one at a time. 
which alternative in the cycle should be elected. This is a fundamental problem in political economy that we show, through this second set of properties, to be addressed simply and naturally by both voting institutions. The two voting procedures approach the problem from the same angle, that is, by systematically identifying an alternative as especially prominent. However, as in the above Condorcet case, they follow logically opposed directions. While the Euro-Latin procedure involves identifying a prioritarian alternative, one that is chosen whenever it is part of a triple of alternatives forming a cycle, the Anglo-American procedure identifies an antiprioritarian alternative, an alternative that is never chosen when it is part of a triple of alternatives forming a cycle, and that identifies the alternative to be selected: precisely that alternative that is preferred by a majority to the antiprioritarian alternative.

The third and last pair of properties imposes consistency requirements in the elections between related sets of alternatives. In both cases, the properties impose structure on the elected outcomes so that the latter do not depend capriciously on the set of alternatives to vote upon.

The rest of the paper is organized as follows. Section 2 formally presents the environment, gives the definitions of the voting procedures, and introduces the equilibrium notion used thereafter. Section 3 is devoted to the characterization of the Euro-Latin procedure, while Section 4 does the same for the Anglo-American procedure. Finally, Section 5 discusses the nature of the properties used in the characterizations of the procedures, shows the independence of the axioms by studying alternative voting institutions, and establishes the connection between our exercise and implementation theory. All the proofs are contained in the Appendix.

\section{Basic Definitions}

Let $X$ be a finite set of $m$ alternatives and let $n$ denote the number of voters. For convenience we assume that $n$ is odd. A decision problem is a pair $(P, A)$, where $P=\left(P_{1}, \ldots, P_{n}\right)$ is a profile of preferences, with each $P_{i}$ being a complete, 
transitive, and asymmetric binary relation on $X$, and $A \subseteq X$ is a set of alternatives to vote for. A decision rule $v$ assigns to each decision problem $(P, A)$ an outcome $v(P, A) \in A .^{3}$

An agenda $\vec{X}=\left(x_{1}, \ldots, x_{m}\right)$ is an ordered list of all the elements in $X$. Given the agenda $\vec{X}$, the associated successive procedure or equivalently the associated Euro-Latin procedure assigns to any set of alternatives $A$, the alternative that survives the following process. The first alternative in $A$ according to the agenda is voted for approval. If the alternative is approved by a majority of individuals, the process stops and this alternative is implemented. If the alternative is rejected, the second alternative of $A$ in the agenda is voted for approval. If the alternative is approved by a majority of individuals, the process stops and this alternative is implemented. Otherwise, the next alternative of $A$ in the agenda is considered, and the process is repeated. If the final alternative in the agenda is reached, it is approved without voting. Consider the extensive game representation of the Euro-Latin procedure restricted to the set of alternatives $A$ when voters have preferences $P$, and denote by $\gamma_{E L}(P, \vec{X}, A)$ its strategic form representation.

Given the agenda $\vec{X}$, the associated amendment procedure or equivalently the associated Anglo-American procedure assigns to any set of alternatives $A$ the alternative that survives the following process. The first pair of alternatives of $A$ in the agenda is voted upon with the one obtaining a majority of votes advancing to the next stage. There, it is paired against the next alternative of $A$ in the agenda, and the process is repeated until the final alternative in the agenda is reached. Consider the extensive game representation of the Anglo-American procedure restricted to the set of alternatives $A$, and denote by $\gamma_{A A}(P, \vec{X}, A)$ its strategic form representation.

Clearly, both procedures are subject to strategic manipulation by sophisticated voters. In the Euro-Latin procedure voters may approve an early alternative in the agenda in order to avoid the selection of a later one. In the Anglo-American procedure voters may pass an alternative only because it can defeat a posterior one, and not because it is preferred to the one with which it is competing. The

${ }^{3}$ Dutta, Jackson, and Le Breton $(2001,2002)$ also consider decision rules in the domain of all the subsets of $X$. 
characterization of the equilibrium outcomes under the two procedures signifies a challenge that we address in this paper.

One drawback with sophisticated behavior is that Nash equilibrium may yield absurd outcomes. For example, in a simple two-alternative setting, if all players vote for the same alternative, independently of their preferences, we have a Nash equilibrium. The refinement that rules out this sort of behavior is the use of undominated strategies. Clearly, in the former two-alternative example, voting for the less preferred alternative is weakly dominated, and hence would be eliminated. It is well known that binary voting procedures like the one we study here are dominance solvable. That is, the iterated elimination of weakly dominated strategies in the strategic form representation leads to a unique Nash equilibrium outcome (see Moulin 1979, McKelvey and Niemi 1978, and Austen-Smith and Banks 2005). The use of Nash equilibrium in undominated strategies is, therefore, standard practice in voting settings like ours, and this is the one we adopt here. We denote by $U N E\left[\gamma_{E L}(P, \vec{X}, A)\right]$ and $U N E\left[\gamma_{A A}(P, \vec{X}, A)\right]$ the corresponding equilibria in undominated strategies of the Euro-Latin and Anglo-American procedures respectively.

\section{Characterization of the Euro-Latin Procedure}

Given a decision problem $(P, A)$, a Condorcet winner is an alternative in $A$ such that, for any other alternative $A$, a majority of voters ranks the former above the latter. In other words, the Condorcet winner majority dominates all other alternatives in $A$. The properties of a Condorcet winner make it highly desirable as the social outcome of any political problem. This leads us to consider the classical Condorcet Consistency property.

Condorcet Consistency (CC). The decision rule selects the Condorcet winner whenever this alternative exists.

It is well known that Condorcet winners do not always exist. The simplest situation in which Cordorcet winners fail to exist involves three alternatives $x, y$ and $z$, with $x$ majority dominating $y, y$ majority dominating $z$, and $z$ majority dominating $x$. We refer to this three-alternative situation as a Condorcet cycle. 
The presence of a Condorcet cycle immediately raises the problem of selecting one alternative from the triple forming the cycle. A possible approach to this problem entails identifying an alternative that for certain reasons is given priority over the rest of the alternatives. This may be so because it represents the status quo, for example. In any event, this alternative is always selected in every Condorcet cycle that includes it. More formally, we say that $a$ is prioritarian in $A$ if, for any preference profile $P$, and for any triple $T_{A}$ of alternatives in $A$ forming a Condorcet cycle that involves alternative $a$, we have $v\left(P, T_{A}\right)=a$.

Condorcet Priority (CP). The decision rule admits a prioritarian alternative for any set of alternatives.

It is advisable to explore in more detail two simple implications of $\mathrm{CP}$ that contribute to a better understanding of the property. First, note that CP implies that for every $A$ with at least three alternatives there is a unique prioritarian alternative. This is easy to see. Suppose on the contrary that $a$ and $b$ are prioritarian in $A$. Then, there exist a preference profile $P$ and alternative $c \in A$ such that $a, b$ and $c$ form a Condorcet cycle. It follows that whatever the outcome from $v(P,\{a, b, c\})$ it leads to a contradiction. Second, it is also immediate that if alternative $a$ is prioritarian in $A$, then $a$ is prioritarian in every $B \subseteq A$ such that $a \in B$. That is, there is a great deal of consistency across menus of options in the determination of prioritarian alternatives.

Let us now consider the third and last characterizing property of the Euro-Latin procedure. Suppose that the collectivity of voters has to select an alternative from a set $A$. A natural process involves dividing the scrutiny of alternatives in $A$ into three stages. In stage 1 the collectivity of voters, say the committee, decides over a subset of $A$, say $B$, in stage 2 the committee decides over the remaining alternatives in $A$, say $C$, and in stage 3 , the committee decides over the selection from $B$ and that from $C$. More formally, we say that a non-empty partition $(B, C)$ of $A$ constitutes a division of $A$ if, for any $D \subseteq A$ and for any preference profile $P, v(P, D)=v(P,\{v(P, D \cap B), v(P, D \cap C)\})$. Notice that in the definition of a division we implicitly admit the possibility that some alternatives in $A$ may be 
dropped in the process.

Division Consistency (DC). The decision rule admits a division for any set of alternatives.

Note that Division Consistency immediately implies that if $(B, C)$ is a division of $A,(B \cap S, C \cap S)$ is a division of $S$, whenever $S$ is contained in $A$. We now show that the three properties described above completely and uniquely characterize the equilibrium outcome of the Euro-Latin procedure.

Theorem 1. A decision rule $v$ satisfies $C C, C P$ and $D C$ if and only if there exists an agenda such that $v$ is the undominated Nash equilibrium outcome of the Euro-Latin procedure.

The intuition of the 'if' part is simple. The 'only if' part of the proof proceeds as follows. Condorcet Priority guarantees that there is a single prioritarian alternative in each set of alternatives. We use this to construct an agenda $\vec{X}$ placing later those alternatives that are prioritarian in bigger sets. We then show that the outcomes of the decision rule coincide with the result in the Anglo-American procedure with agenda $\vec{X}$, when voters vote sincerely, that is when voters truthfully reveal their actual preferences. In order to do so we prove that for any set of alternatives $A$ and a prioritarian alternative $h$ of $A$, the sets $A \backslash\{h\}$ and $\{h\}$ form the unique division of $A$. That is, we show that the prioritarian alternative of a set can always be separated from the set, without further consequences for the selection. Finally we use the well-known relationship by which the result of sincere voting in an Anglo-American procedure with agenda $\vec{X}$ is exactly the Nash equilibrium in undominated strategies of the Euro-Latin procedure with the agenda following the opposite order to the one in $\vec{X}$ (see Miller 1977). Then, we conclude that for the Euro-Latin procedure the construction of the agenda places the prioritarian alternative in $X, x_{1}$, first in the ordered list, followed by the prioritarian alternative in $X \backslash\left\{x_{1}\right\}$, and so on. Intuitively, therefore, under sophisticated voting in the Euro-Latin procedure, the strongest alternatives, those that are selected in each Condorcet cycle, are considered first in the agenda. 


\section{Characterization of the Anglo-American Procedure}

Given a decision problem $(P, A)$, a Condorcet loser is an alternative in $A$ such that, for any other alternative $A$, a majority of voters places the former below the latter. Notice that the Condorcet loser, whenever it exists, is unique. The Condorcet loser is a highly undesirable alternative and hence not only would it be absurd to select it as the outcome of a decision problem, but also it is natural that any sensible voting procedure be robust to the presence or absence of such an alternative. This leads us to the following consistency property. ${ }^{4}$

Condorcet Loser Consistency (CLC). $v(P, A)=v(P, A \backslash\{a\})$ whenever $a$ is a Condorcet loser in $(P, A)$.

In the presence of a Condorcet cycle in the triple $\{x, y, z\}$, our first approach above identifies an element that is given priority. An alternative approach consists in identifying an alternative that is never prioritized in such situations. This may encourage changes if the alternative is the default one, or avoid risky outcomes when decisions are unclear or controversial. More formally, we say that $a$ is antiprioritarian in $A$ if, for any preference profile $P$, and for any triple $T_{A}$ in $A$ that forms a Condorcet cycle involving alternatives $a$ and $x$, we have $v\left(P, T_{A}\right)=x$ if and only if $v(P,\{x, a\})=x$, with $x \neq a$. That is, alternative $a$ is antiprioritarian if (i) it is never selected when being part of a Cordorcet cycle $T_{A}$, and (ii) the alternative selected from $T_{A}$ is precisely the one that is preferred to $a$ by the majority. That is, the alternative $a$ that is considered as antiprioritarian identifies in each case the alternative to be selected.

Condorcet Antipriority (CA). The decision rule admits an antiprioritarian alternative for any set of alternatives.

As in the case of prioritarian alternatives, in every $A$ with $|A| \geq 3$, there is a unique antiprioritarian alternative, and also if $a \in B \subseteq A$ is antiprioritarian in $A$,

\footnotetext{
${ }^{4}$ Indeed, in the Discussion section below we argue that Euro-Latin and Anglo-American procedures satisfy both this consistency property and Condorcet Consistency.
} 
$a$ is also antiprioritarian in $B$. One relevant distinction in the structure of the two properties is the relevance of the direction of Condorcet cycles. For Condorcet Priority, if alternative $a$ is prioritarian in $A$ and $a$ is part of a Condorcet cycle $T_{A}$ in $A$, the direction of the cycle is immaterial for the outcome from $v\left(P, T_{A}\right)$. For Condorcet Antipriority the direction of the cycle is however relevant. Clearly, if $a$ is antiprioritarian and majority preferred to $b, b$ majority preferred to $c$, and $c$ majority preferred to $a$ the outcome from $\{a, b, c\}$ is option $c$. However, if the direction of the cycle is the opposite, then the outcome is $b$.

Our final property establishes a consistency requirement across sets of alternatives. Having identified one antiprioritarian alternative in a set $A$, one should be able to remove those alternatives dominated by the antiprioritarian one, since one can argue that they are not natural candidates for election, and concentrate on the rest. The election in this subset must coincide with the election in the original set.

Elimination Consistency (EC). If $a$ is an antiprioritarian alternative in $A$ and $v(P,\{a, y\})=a$, then $v(P, A)=v(P, A \backslash\{y\})$.

We now show that Condorcet Loser Consistency, Condorcet Antipriority and Elimination Consistency completely and uniquely characterize the equilibrium outcome of Anglo-American procedures.

Theorem 2. A decision rule $v$ satisfies $C L C, C A$ and $E C$ if and only if there exists an agenda such that $v$ is the undominated Nash outcome of the AngloAmerican procedure.

We now provide the intuition for the 'only if.' We first construct the agenda $\vec{X}$ as follows. Consider first the antiprioritarian alternative $x_{1}$ in $X$, and place it at the end of the agenda. Then, consider the antiprioritarian alternative $x_{2}$ in $X \backslash\left\{x_{1}\right\}$ and place it in the second to last position in the agenda, and so on. If we interpret antiprioritarian alternatives as the most controversial ones, this means that the Anglo-American procedure places these alternatives at the very end of the agenda. 
The second step in the proof involves showing that for every $P$ and $A$, the election from a decision rule satisfying the properties is the limit $x^{*}$ of a sequence of stepping stones in the agenda. This sequence is formed by: (i) the last alternative of $A$ according to the agenda $\vec{X}$, (ii) the last alternative of $A$ that majority dominates the alternative in (i), (iii) the last alternative of $A$ that majority dominates the alternatives in (i) and (ii), etc. The proof of this step uses the following idea. The last alternative is antiprioritarian, and thus, by EC and CLC, we can concentrate exclusively on those alternatives that dominate it. A recursive argument concludes the proof. Finally, we show through an inductive argument, along the lines of Shepsle and Weingast (1984), that the $U N E\left[\gamma_{A A}(P, \vec{X}, A)\right]$ is exactly the limit of this sequence.

\section{Discussion}

\subsection{Comments on the Axiomatic Structures of the Euro-Latin and} Anglo-American Procedures. We have shown that while Euro-Latin procedures are fully characterized by Condorcet Consistency, Condorcet Priority and Division Consistency, Anglo-American procedures are fully characterized by Condorcet Loser Consistency, Condorcet Antipriority, and Elimination Consistency. It is illuminating to note the shared structure of the two systems of properties characterizing the two voting procedures.

First, both Condorcet Consistency for Euro-Latin procedures and Condorcet Loser Consistency for Anglo-American procedures follow the fundamental principle of Condorcet-type reasoning. While the former imposes selecting the alternative that majority dominates all others whenever it exists, the latter imposes that the outcome should not depend on the presence of the alternative that is majority dominated by all others, whenever it exists. Furthermore, given the desirability of the two properties, one may wonder whether Condorcet Loser Consistency is satisfied by Euro-Latin procedures and the same is true for Condorcet Consistency and Anglo-American procedures. The answer to both questions is clearly yes. In fact we can prove that we can replace Condorcet Consistency by Condorcet Loser Consistency in our characterization of the Euro-Latin procedure. When Division Consistency holds, then both properties are indeed equivalent. 
Proposition 1. A decision rule $v$ satisfying $D C$ satisfies $C C$ if and only if it satisfies CLC.

Second, properties Condorcet Priority in the Euro-Latin case and Condorcet Antipriority in the Anglo-American case again follow the same type of logic, with opposite directions. Both properties apply in the presence of cycles, and while the former identifies an alternative that gains prevalence whenever it is present, the latter identifies an alternative as unchoosable. These two properties shape the order of the agenda. While in the Euro-Latin case the prioritarian alternatives come first in the agenda, in the Anglo-American case the antiprioritarian alternatives come last in the agenda.

Finally, Division Consistency and Elimination Consistency both impose structure on the outcomes selected by the voting procedures across sets of alternatives.

\subsection{Other Voting Procedures and the Independence of the Axioms.}

We now suggest other voting institutions than the Anglo-American and EuroLatin ones, with the purpose of exploring the consequences of relaxing each of the properties we have studied, one at a time. This illustrates the power of the axioms, that is, the structure they are imposing, and at the same time it shows the independence of the properties.

Consider first a decision rule that completely ignores the preferences of the voters, and follows some external order over the alternatives, say a virtue book. More specifically, the virtue book establishes a linear order over the set of alternatives and the decision rule selects in each decision problem the maximal alternative according to the book. The rule trivially satisfies Condorcet Priority, the prioritarian alternative in a set being the most virtuous alternative in the set. Also, given the maximizing nature of the rule, Division Consistency holds since any division of the set of alternatives is inessential for the final selection. However, since the virtue book completely disregards the views of individuals, it might be the case that all individuals in society prefer the least virtuous alternative in a set to the most virtuous one and yet the rule chooses the latter alternative, violating Condorcet Consistency.

Alternatively, a modified version of the virtue decision rule would aim to block the least virtuous alternative, and in so doing would attend the views of the 
individuals by selecting the alternative that presents the highest resistance to it. That is, the modified virtue book rule selects the alternative with the largest margin with respect to the least virtuous alternative (and, say, it respects the selection of the virtue book in the event of a tie). The rule satisfies Condorcet Antipriority. To see this, note that the antiprioritarian alternative in a set is the least virtuous alternative. Then, in the event of a cycle, it follows immediately that the alternative that dominates the antiprioritarian one has a larger margin than the other alternative. The rule also satisfies Elimination Consistency since no alternative is dominated by the least virtuous one. However, the rule fails to satisfy Condorcet Loser Consistency since it may be the case that the Condorcet loser is the alternative with the largest margin to the least virtuous one, and hence it would be selected by the modified virtue decision rule.

More democratic versions of the above two rules would involve attending the views of society when these are consistent with Condorcet notions. The Condorcet virtue book would select the Condorcet winner whenever it exists, and would follow the virtue book rule otherwise. This rule trivially satisfies Condorcet Consistency. It also satisfies Condorcet Priority since the virtue book is applied in the presence of cycles. However, it fails to satisfy Division Consistency. To see this, consider a three-element set, and following a similar analysis to the one adopted in the proof of Theorem 1, note that the only possible division involves the separation of the prioritarian alternative from among the other two. It might be the case that there is a cycle involving the three alternatives and hence the prioritarian alternative is selected in the three-element set, and that the alternative selected from among the non-prioritarian ones majority dominates the prioritarian, causing the latter not to be selected in the three-stage process, thus violating Division Consistency.

The Condorcet modified virtue book would similarly rule out Condorcet losers, applying successively the property of Condorcet Loser Consistency, whenever this is possible, and behaving like the modified virtue book when Condorcet losers do not exist. This, apart from obviously satisfying Condorcet Loser Consistency, satisfies Condorcet Antipriority since the modified virtue book is applied in the presence of cycles. However, it violates Elimination Consistency. Consider a set of alternatives $\{a, y, z\}$ over which $a$ is the antiprioritarian alternative and 
suppose that a majority dominates alternative $y$, and $y$ dominates $z$. It might be the case that, by focussing on the largest margin against $a$, the rule selects alternative $y$, violating Elimination Consistency.

Finally, consider a binary game tree where (i) all alternatives appear once as terminal nodes of the tree and (ii) there exists at least one node with two nonterminal successors. ${ }^{5}$ Define, then, the generalized agenda rule as the rule that selects the undominated Nash equilibrium of the normal form game induced by the binary game. This rule obviously satisfies Condorcet Consistency and Condorcet Loser Consistency. Now, consider one of the special nodes in point (ii) and four alternatives that are successors of it, two from each of the two non-terminal successors. It is immediate to see that for any subset of three alternatives involving a Condorcet cycle, the selection of the rule is the alternative that branches away on its own from the other two. Consequently, there is no prioritarian alternative in the set of four alternatives. Hence, Condorcet Priority does not hold in general. A similar reasoning shows that Condorcet Antipriority is also violated. Finally, the rule satisfies Division Consistency by the equilibrium nature of the rule and Elimination Consistency since no antiprioritarian alternative exists.

5.3. Connection to Implementation Theory. We would like to note here that our exercise can be related to classical implementation theory. ${ }^{6}$ In the language of implementation, our exercise might be restated as follows. Decision rules satisfying $\mathrm{CC}, \mathrm{CP}$ and DC, or alternatively satisfying CLC, CA and EC can be implementable via the undominated Nash solution. In particular, our analysis shows that we can use simple mechanisms for the implementation, since the former is implemented using the Euro-Latin procedure and the latter by the Anglo-American procedure.

The main difference between our approach and the one typically used in implementation theory is that, in our case, a decision rule is defined not only over all possible preference profiles but also over all possible subsets of alternatives, while implementation theory typically works over the grand set of alternatives only.

\footnotetext{
${ }^{5}$ Some of the conclusions on the independence of the axioms require at least four alternatives, such as the argument that follows.

${ }^{6}$ See Jackson (2001) for a survey on implementation theory.
} 


\section{Appendix A. Proofs}

Proof of Theorem 1: We first prove that the Nash equilibrium in undominated strategies of a Euro-Latin procedure satisfies the properties. Clearly, CC is immediate. To check CP, we just need to observe that the first alternative of the set according to the agenda is prioritarian. The reason for this is that, if such an alternative is involved in a Condorcet cycle, its rejection would imply the election of the majoritarian winner alternative from among the other two. Because of the Condorcet cycle structure, that alternative is majority inferior to the first one. Finally, any set of alternatives admits a division in which all the alternatives but the first one, according to the agenda, are separated from the first alternative. We now prove the converse statement using a lemma.

Lemma 1. For any $A$ with $|A| \geq 3$ let $h$ be the prioritarian alternative of $A$, then $(A \backslash\{h\},\{h\})$ is the unique division of $A$.

Proof of Lemma 1: We proceed by contradiction. Suppose that $(B, C)$ is a division of $A$, with $h \in B$ and $|B| \geq 2$. Let $b \in B \backslash\{h\}$ and $c \in C$ and consider a profile $P$ where: (i) alternatives $b, c$ and $h$ are preferred to any other alternative in $A$ by every individual, and (ii) alternatives $b, c$ and $h$ form a Condorcet cycle such that $c$ is majority preferred to $h, h$ to $b$ and $b$ to $c$. By DC, since $\{b, h\}$ is a subset of $B$ and $\{c\}$ is a subset of $\mathrm{C}$, we have $v(P,\{b, c, h\})=v(P,\{v(P,\{b, h\}), c\})$. Given the structure of $P$, CC implies $v(P,\{b, h\})=h$ and $v(P,\{b, c, h\})=v(P,\{h, c\})=c$. This is a clear contradiction with $h$ being prioritarian in $A$.

Now we define an agenda $\vec{X}$. By CP, there exists a prioritarian alternative in $X$. Let $x_{n}$ be such an alternative and let it be placed at the end of the agenda. Recursively, given $x_{k+1}, \ldots, x_{n}$, we can use CP to define $x_{k}$ as a prioritarian alternative of $X \backslash\left\{x_{k+1}, \ldots, x_{n}\right\}$.

Next, we prove that $v$ is the outcome of Anglo-American voting over the agenda $\vec{X}$ when voters vote sincerely, that is truthfully reporting their preferences. We prove it by induction on the cardinality of set $A$. By CC, if $|A|=2$, the decision rule selects the majoritarian alternative, and hence the claim follows. Suppose the claim is true for any set of alternatives with cardinality lower than or equal 
to $p \geq 2$. We now prove that the claim is true for any set of alternatives $A$ with $|A|=p+1$. Let $x_{m}$ be the last element of $A$ in the agenda. Given the induction hypothesis, $v\left(P, A \backslash\left\{x_{m}\right\}\right)$ is the outcome of sincere voting in the Anglo-American procedure $\gamma_{A A}\left(P, \vec{X}, A \backslash\left\{x_{m}\right\}\right)$. Clearly, by CC $v\left(P,\left\{v\left(P, A \backslash\left\{x_{m}\right\}\right), x_{m}\right\}\right)$ is the outcome of sincere voting in the Anglo-American procedure restricted to this pair of alternatives. Given that $x_{m}$ is the last element of $A$, it is straightforward that $v\left(P,\left\{v\left(P, A \backslash\left\{x_{m}\right\}\right), x_{m}\right\}\right)$ is also the outcome of sincere voting for the AngloAmerican procedure $\gamma_{A A}(P, \vec{X}, A)$. To conclude the claim, we only need to show that $v\left(P,\left\{v\left(P, A \backslash\left\{x_{m}\right\}\right), x_{m}\right\}\right)=v(P, A)$. By construction, $x_{m}$ is the prioritarian alternative of $\left\{x_{1}, x_{2}, \ldots, x_{m}\right\}$ and hence it is also the prioritarian alternative of $A$. Hence, by Lemma 1 we know that $\left(A \backslash\left\{x_{m}\right\},\left\{x_{m}\right\}\right)$ is the only division of $A$ and hence $v\left(P,\left\{v\left(P, A \backslash\left\{x_{m}\right\}\right), x_{m}\right\}\right)=v(P, A)$.

To conclude the proof, we use the well-known result on the equivalence between the outcomes of sincere voting in Anglo-American procedures with a given agenda $\vec{X}$ and the outcome of the Nash equilibrium in undominated strategies of the Euro-Latin procedure where the order of the agenda $\vec{X}$ is reversed, given the preference profile $P$ (see Miller 1977; see also Moulin 1979).

Proof of Theorem 2: We first prove that the Nash equilibrium in undominated strategies of an Anglo-American procedure satisfies the properties. CLC is immediate. To check CA, we just need to observe that the last alternative of the set according to the agenda is antiprioritarian. Suppose there exists a Condorcet cycle involving alternatives $x, y$ and $a$, where $x$ majority dominates $y, y$ majority dominates the last alternative in the triple $a$ and $a$ majority dominates $x$. Since the outcomes from $\{x, a\}$ and $\{y, a\}$ are $a$ and $y$ respectively, and $y$ majority dominates $a$, the outcome of the first election, namely between $x$ and $y$, will be $y$, which will be confirmed in the final election. Hence, $a$ is antiprioritarian, as announced. Finally, we prove that the Nash equilibrium in undominated strategies of an Anglo-American procedure satisfies EC. The claim is trivial for sets involving two alternatives. Notice that for any set $A$ with at least three alternatives, the antiprioritarian alternative must be the last alternative of $A$ in the agenda, say $a$. This follows immediately from our previous argument on CA. Let $y$ be majority dominated by $a$. Clearly, $y$ cannot be the selected alternative 
in $A$ since $y$ reaching the final election against $a$ would entail the election of $a$. Then, it follows that at equilibrium the outcome from $A$ must be the same as the outcome from $A \backslash\{y\}$, as desired. We now prove the converse statement.

Let $v$ satisfy CLC, CA and EC. We first construct an agenda $\vec{X}$. By CA, there exists one alternative which is antiprioritarian in $X$. Denote it by $x_{1}$. Suppose we have defined $x_{1}, \ldots, x_{k}$. By CA, there exists one alternative which is antiprioritarian in $X \backslash\left\{x_{1}, \ldots, x_{k}\right\}$. Denote it by $x_{k+1}$. This process defines an ordered list of alternatives $x_{1}, \ldots, x_{n}$. We construct the agenda by setting $\vec{X}=\left(x_{n}, x_{n-1}, \ldots, x_{1}\right)$.

Now, given the agenda $\vec{X}$, we associate with each pair $(P, A)$ a sequence of alternatives in $A, t_{1}(P, A), t_{2}(P, A), \ldots$ as follows. $t_{1}(P, A)$ is the last alternative in $A$ according to the agenda $\vec{X} . t_{2}(P, A)$ is the last alternative in $A$, according to the agenda $\vec{X}$, that majority dominates alternative $t_{1}(P, A)$, if it exists. Otherwise, $t_{2}(P, A)=t_{1}(P, A)$. Given $t_{1}(P, A), \ldots, t_{s}(P, A)$, denote by $t_{s+1}(P, A)$ the first alternative from $A$ in the agenda $\vec{X}$ that majority dominates all alternatives $t_{1}(P, A), \ldots, t_{s}(P, A)$, if it exists. Otherwise, $t_{s+1}(P, A)=t_{s}(P, A)$.

Lemma 2. Given $A$ and $P, v(P, A)=\lim t_{i}(P, A)$.

Proof of Lemma 2: Notice that by construction, $t_{1}(P, A)$ is antiprioritarian in $A=A_{1}$. Thus, by EC, $v\left(P, A_{1}\right)=v\left(P, A_{1}^{\prime}\right)$, where $A_{1}^{\prime}$ contains exactly the alternative $t_{1}(P, A)$ and the set of alternatives that are majority dominated by $t_{1}(P, A)$ in $A_{1}$. If $A_{1}^{\prime}=\left\{t_{1}(P, A)\right\}$, we are done. Otherwise, $t_{1}(P, A)$ is a Condorcet loser in $A_{1}^{\prime}$ by construction. By CLC, $v\left(P, A_{1}^{\prime}\right)=v\left(P, A_{2}\right)$ where $A_{2}=A_{1}^{\prime} \backslash\left\{t_{1}(P, A)\right\}$. By construction, $t_{2}(P, A)$ is the last alternative in $A_{2}$ according to the agenda $\vec{X}$. Hence, $t_{2}(P, A)$ is antiprioritarian in $A_{2}$, and by EC, $v\left(P, A_{2}\right)=v\left(P, A_{2}^{\prime}\right)$ where $A_{2}^{\prime}$ is the set of alternatives that are majority dominated by $t_{2}(P, A)$ in $A_{2}$, and that contains $t_{2}(P, A)$. If $A_{2}^{\prime}=\left\{t_{2}(P, A)\right\}$, we are done. Otherwise, $t_{2}(P, A)$ is a Condorcet loser in $A_{2}^{\prime}$ by construction. Then, again $t_{3}(P, A)$ is the last alternative in $A_{3}=A_{2}^{\prime} \backslash\left\{t_{2}(P, A)\right\}$ according to the agenda and given the finiteness of $X$, the iteration of this process proves the lemma.

Lemma 3. Given $A$ and $P, U N E\left[\gamma_{A A}(P, \vec{X}, A)\right]=\lim t_{i}(P, A) .^{7}$

${ }^{7}$ Shepsle and Weingast (1984) proved an analogous result. We include Lemma 3 here for completeness. 
Proof of Lemma 3: We prove this lemma by induction on the cardinality of $A$. If $A$ contains two alternatives, the claim follows immediately. Suppose that the claim is true for sets containing up to $k$ alternatives, and consider $A$ with $k+1$ alternatives. Take the first and second alternatives in $A$ with respect to the agenda, say $x$ and $y$. If the alternative $x$ (resp. $y$ ) is voted off, then by the induction hypothesis, the outcome of the equilibrium in undominated strategies is the limit $l_{1}=\lim t_{i}(P, A \backslash\{x\})$ (resp., $l_{2}=\lim t_{i}(P, A \backslash\{y\})$ ). By construction, the outcome of the Nash equilibrium in undominated strategies in $A$ is the alternative that is majority preferred among these two alternatives, $l_{1}$ and $l_{2}$. We now show that this coincides with the $\lim t_{i}(P, A)$. We distinguish between the following cases:

- $l_{1} \neq y$ and $l_{2} \neq x$. Then $l_{1}=l_{2}$ and this element is different from $x$ and $y$. By construction of $l_{1}, l_{1}$ is majority preferred to both $x$ and $y$. Hence, $l_{1}$ is the limit of the sequence associated with $A$.

- $l_{1}=y, l_{2} \neq x$. By construction, $y$ majority dominates $l_{2}$ and hence $y$ is the outcome of the Nash equilibrium in undominated strategies in $A$. But clearly, $y$ belongs to the sequence in $A$, and since $x$ does not belong to the sequence in $A \backslash\{y\}$, it does not belong to the sequence in $A$ either. Hence, $y$ is the limit of the sequence of $A$, as desired.

- $l_{1} \neq y, l_{2}=x$. By construction, $x$ majority dominates $l_{1}$ and hence $x$ is the outcome of the Nash equilibrium in undominated strategies in $A$. But clearly, $y$ does not belong to the sequence in $A$ since $y$ does not belong to the sequence in $A \backslash\{x\}$. As a consequence, $x$ belongs to it, and it is the limit of the sequence in $A$.

- $l_{1}=y$ and $l_{2}=x$. The outcome of the Nash equilibrium in undominated strategies in $A$ is the alternative in $\{x, y\}$ that majority dominates the other. But notice that $y$ belongs to the sequence in $A$, and $x$ is in the sequence if and only if $x$ majority dominates $y$. Hence, the limit of the sequence is the alternative in $\{x, y\}$ that majority dominates the other, as desired.

The two lemmata together conclude the proof. 
Proof of Proposition 1: We first prove that a decision rule $v$ satisfying DC and $\mathrm{CC}$ also satisfies CLC. Consider $(P, A)$ and suppose that the Condorcet loser $c l(P, A)$ exists. We need to prove that $v(P, A)=v(P, A \backslash\{c l(P, A)\})$. We define iteratively a finite family of nested sets $A_{i}$ each containing the Condorcet loser $c l(P, A)$. Let $A_{1}=A$. Given the sets $A_{1}, A_{2}, \ldots, A_{i}$ containing $c l(P, A)$, by DC, there exists a division of $A_{i}, B_{i}$ (which without loss of generality we assume to contain $\operatorname{cl}(P, A))$ and $C_{i}$. Define $A_{i+1}$ as $B_{i}$ whenever $B_{i}$ contains some alternative in addition to the Condorcet loser. Otherwise, the family stops at $A_{i}$.

Now, from the constructed family, we know that:

(1) $v\left(P, A_{i}\right)=v\left(P,\left\{v\left(P, B_{i}\right), v\left(P, C_{i}\right)\right\}\right)$ and

(2) $v\left(P, A_{i} \backslash\{c l(P, A)\}\right)=v\left(P,\left\{v\left(P, B_{i} \backslash\{\operatorname{cl}(P, A)\}\right), v\left(P, C_{i}\right)\right\}\right)$.

Hence, $v\left(P, A_{i}\right)=v\left(P, A_{i} \backslash\{c l(P, A)\}\right)$ if $v\left(P, B_{i}\right)=v\left(P, B_{i} \backslash\{c l(P, A)\}\right)$. That is, $v\left(P, A_{i}\right)=v\left(P, A_{i} \backslash\{c l(P, A)\}\right)$ if $v\left(P, A_{i+1}\right)=v\left(P, A_{i+1} \backslash\{c l(P, A)\}\right)$. The conjunction of all these relationships leads to $v(P, A)=v(P, A \backslash\{c l(P, A)\})$ if $v\left(P, A_{k}\right)=v\left(P, A_{k} \backslash\{c l(P, A)\}\right)$, where $A_{k}$ is the last set in the family. In this case, $B_{k}=\{\operatorname{cl}(P, A)\}$ and given that $\operatorname{cl}(P, A)$ is majority dominated by all alternatives in $A_{k} \backslash\{c l(P, A)\}$ we have that $v\left(P, A_{k}\right)=v\left(P,\left\{v\left(P, B_{k}\right), v\left(P, C_{k}\right)\right\}\right)=$ $v\left(P,\left\{c l(P, A), v\left(P, A_{k} \backslash\{c l(P, A)\}\right)\right\}\right)=v\left(P, A_{k} \backslash\{c l(P, A)\}\right)$, as desired.

We now prove that a decision rule $v$ satisfying DC and CLC also satisfies CC. We prove it by induction on the cardinality of the set of alternatives. Suppose that the set has two alternatives. Then, the Condorcet winner is selected by direct application of CLC. Now suppose that, if they exist, Condorcet winners are always selected for the corresponding sets, which are supposed to have cardinality smaller than or equal to $k$. Let $(P, A)$ with cardinality of $A$ be equal to $k+1$ and suppose that the Condorcet winner $c w(P, A)$ exists. By DC, there exists a division $(B, C)$ of $A$. Suppose without loss of generality that $c w(P, A) \in B$. Hence, $v(P, A)=v(P, B \cup C)=v(P,\{v(P, B), v(P, C)\})$. Since $c w(P, A)=$ $c w(P, B)=c w(P, v(P, C) \cup\{c w(P, A)\})$, by the inductive hypothesis we know that $v(P,\{v(P, B), v(P, C)\})=v(P,\{c w(P, A), v(P, C)\})=c w(P, A)$. Hence, $v(P, A)=c w(P, A)$, and CC holds. 


\section{REFERENCES}

[1] Apesteguia, J., M.A. Ballester, and R. Ferrer (2011), "On the Justice of Decision Rules," Review of Economic Studies, 78:1-16.

[2] Austen-Smith, D and J.S. Banks (2005), Positive Political Theory II: Strategy and Structure,. University of Michigan Press.

[3] Banks, J.S. (1985), "Sophisticated Voting Outcomes and Agenda Control," Social Choice and Welfare, 1:295-306.

[4] Bag P.K., H. Sabourian, and E. Winter (2009), "Multi-stage Voting, Sequential Elimination and Condorcet Consistency," Journal of Economic Theory, 144:1278-1299.

[5] Bernheim, B.D., A. Rangel, and L. Rayo (2006), "The Power of the Last Word in Legislative Policy Making," Econometrica, 74:1161-1190.

[6] Black, D. (1948), "On the Rationale of Group Decision-making," Journal of Political Economy, 56:23-34

[7] Dutta, B., M.O. Jackson, M. Le Breton (2001), "Strategic Candidacy and Voting Procedures," Econometrica, 69:1013-1037.

[8] Dutta, B., M.O. Jackson, M. Le Breton (2002), "Voting by Successive Elimination and Strategic Candidacy," Journal of Economic Theory, 103:190-218.

[9] Eckel, C. and C.A. Holt (1989), "Strategic Voting in Agenda-Controlled Committee Experiments," American Economic Review, 79:763-773.

[10] Farquharson, R (1969) Theory of Voting. New Haven: Yale University Press.

[11] Ferejohn, J., M. Fiorina, R.D. McKelvey (1987), "Sophisticated Voting and Agenda Independence in the Distributive Politics Setting," American Journal of Political Science, 31:169-193.

[12] Jackson, M.O. (2001), "A Crash Course in Implementation Theory," Social Choice and Welfare, 18:605-708.

[13] McKelvey, R.D. (1981), "A Theory of Optimal Agenda Design," Management Science, $27: 303-321$.

[14] McKelvey, R.D. and R.G. Niemi (1978), "A Multistage Game Representation of Sophisticated Voting for Binary Procedures," Journal of Economic Theory, 18:1-22.

[15] Miller, N.R. (1977), "Graph-Theoretical Approaches to the Theory of Voting," American Journal of Political Science, 21:769-803.

[16] Miller, N.R. (1980), "A New Solution Set for Tournaments and Majority Voting: Further Graph- Theoretical Approaches to the Theory of Voting," American Journal of Political Science, 24:68-96.

[17] Moser, S. (2007), "A Comparison of Agenda Procedures," mimeo.

[18] Moulin, H. (1979), "Dominance Solvable Voting Schemes," Econometrica, 47:1337-1351.

[19] Moulin, H. (1986), "Choosing from a Tournament," Social Choice and Welfare, 3:271-291. 
[20] Ordeshook P. and T. Schwartz (1987), "Agendas and the Control of Political Outcomes," American Political Science Review, 81:179-99.

[21] Plott, C.R. and M.E. Levine (1978), "A Model of Agenda Influence on Committee Decisions," American Economic Review, 68:146-160.

[22] Rasch, B.R. (2000), "Parliamentary Floor Voting Procedures and Agenda Setting in Europe," Legislative Studies Quarterly, 25:3-23.

[23] Riker, W.H. (1958), "The Paradox of Voting and Congressional Rules for Voting on Amendments," American Political Science Review, 52:349-366.

[24] Shepsle, K.A. and B.R. Weingast (1982), "Institutionalizing Majority Rule: A Social Choice Theory with Policy Implications," American Economic Review, 72:367-371.

[25] Shepsle, K.A. and B.R. Weingast (1984), "Uncovered Sets and Sophisticated Voting Outcomes with Implications for Agenda Institutions," American Journal of Political Science, 28:49-74.

[26] Schwartz, T. (2008), "Parliamentary Procedure: Principal Forms and Political Effects," Public Choice, 136:353-377. 